\title{
IMPACT OF ACTIVE WARMING IN PERIOPERATIVE HEAT LOSS IN LAPAROSCOPIC CHOLECYSTECTOMY
}

\author{
Gabriela Gonçalves, Carla Couto, Marta Pacheco, Ana Veiga de Sá, Carla Cavaleiro, Humberto Machado \\ Centro Hospitalar do Porto, Department of Anaesthesiology and Intensive Care, Porto, Portugal
}

\section{BACKGROUND}

Perioperative heat loss (PHL) is common in patients undergoing surgical procedures, which is associated with many adverse outcomes.

Although active warming techniques (WT) are effective measures for PHL prevention, some studies conclude that short duration surgeries don't seem to benefit with active warming.

\section{GOAL OF THE STUDY}

Evaluate the effect of active warming in preventing perioperative heat loss during Elective Laparoscopic Cholecystectomy.

\section{METHODS}

- Prospective audit.

- Patients undergoing Elective Laparoscopic Cholecystectomy, at our institution, from September to December 2016.

- WT with forced-air warming blankets compared with the same procedures under no active warming technique (nWT), from a previous audit.

- Auricular temperature monitored at five moments:

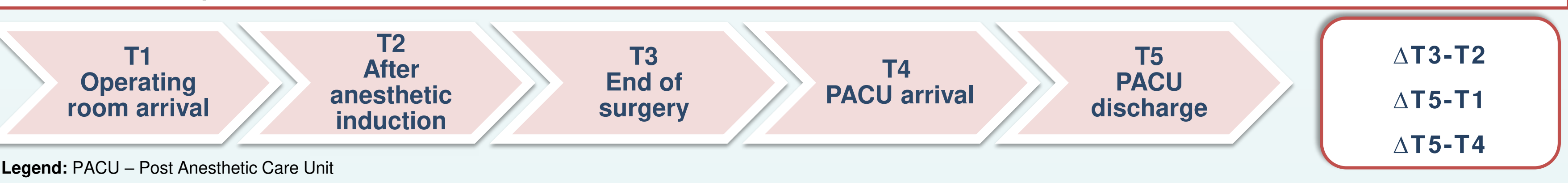

- Gender, Age, American Society of Anaesthesia Physical Status (ASA), Body Mass Index (BMI), Type of anaesthesia, Duration of surgery length were collected.

- Descriptive statistical analysis and Qui Quadrado was runned $(p<0,05)(S P S S \circledast v .22)$.

- Results presented as number, percentage, mean \pm standard deviation (SD) and median.

\section{RESULTS}

Table 1 - Temperature variation at different moments

\begin{tabular}{cccc}
\hline HEAT LOSS & WT $(n=27)$ & $n W T(n=67)$ & $p$ \\
\hline $\begin{array}{c}\Delta T 3-T 2 \\
(m e a n \pm S D)\end{array}$ & $-0,32 \pm 0,76$ & $-0,43 \pm 0,42$ & 0,34 \\
$\begin{array}{c}\Delta T 5-T 1 \\
(m e a n \pm S D)\end{array}$ & $-0,27 \pm 0,74$ & $-0,53 \pm 0,48$ & 0,03 \\
$\begin{array}{c}\Delta T 5-T 4 \\
(m e a n \pm S D)\end{array}$ & $0,57 \pm 0,69$ & $0,35 \pm 0,45$ & 0,51 \\
\hline
\end{tabular}

Table 2 - Temperature variation T5-T1

\begin{tabular}{ccc}
\hline & $W T(n=27)$ & $n W T(n=67)$ \\
\hline $\begin{array}{c}<-0,5^{\circ} \mathrm{C} \\
(n ;[\%])\end{array}$ & $8[30 \%]$ & $35[52 \%]$ \\
$\begin{array}{c}>=-0,5^{\circ} \mathrm{C} \\
(\mathrm{n} ;[\%])\end{array}$ & $18[67 \%]$ & $28[42 \%]$ \\
\hline
\end{tabular}

Table 3 - Patients distribution by gender, age, ASA, IMC, type of anaesthesia and duration of surgery

\begin{tabular}{|c|c|c|}
\hline & WT $(n=27)$ & nWT $(n=67)$ \\
\hline Gender (M/F) & $8(30 \%) / 19(70 \%)$ & $14(21 \%) / 53$ (79\%) \\
\hline Age (median; [min-max]) & 62 [31-88] & 58 [26-87] \\
\hline ASA I/II/III/IV & $\begin{array}{c}2(7 \%) / 16(59 \%) / \\
8(30 \%) / 1(4 \%)\end{array}$ & $\begin{array}{c}9(13 \%) / 47(70 \%) / \\
10(15 \%) / 1(1 \%)\end{array}$ \\
\hline $\mathrm{BMI} \mathrm{Kg} / \mathrm{m}^{2}($ mean $\pm \mathrm{SD})$ & $26,67 \pm 5,30$ & $28,25 \pm 4,39$ \\
\hline Type of anesthesia (GA) & $27(100 \%)$ & $67(100 \%)$ \\
\hline $\begin{array}{l}\text { Duration of surgery (min) } \\
\text { (mean } \pm \text { SD) }\end{array}$ & $48,48 \pm 24,10$ & $48,85 \pm 31,12$ \\
\hline $\begin{array}{l}\text { Length of hospital stay } \\
\text { (mean } \pm \text { SD) }\end{array}$ & $3,35 \pm 4,58$ & $3,10 \pm 4,66$ \\
\hline
\end{tabular}

Legend: BMI - body mass index; GA - General Anesthesia

\section{CONCLUSION}

In this audit, active warming for Elective Laparoscopic Cholecystectomy seems to influence the heat loss associated with surgical procedure, suggesting that forced-air warming may be beneficial. The findings underline the importance of developing new strategies and methods to minimize perioperative heat loss.

\section{REFERENCES}

- Sessler, Daniel I.; Complications and Treatment of Mild Hypothermia; Anesthesiology 2001; 95:531-43

- Reynolds, Luke; Beckmann, James; Kurz, Andrea; Perioperative complications of hypothermia; Best Practice \& Research Clinical Anaesthesiology: Vol. 22, No. 4, pp. 645-657, 2008 